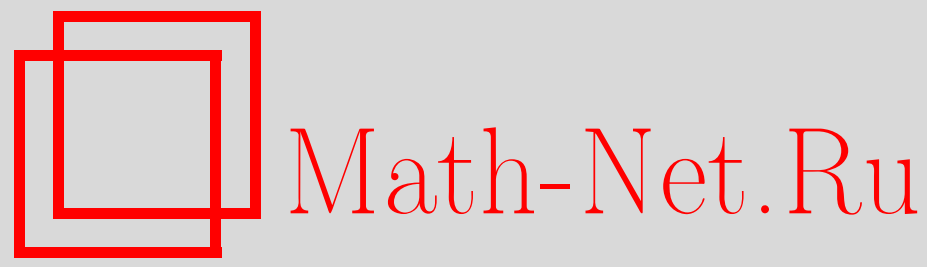

Б. А. Рогозин, Предельная теорема для отношений функций концентрации, Теория вероятн. и ее примен., 2001, том 46, выпуск 4, 801-803

DOI: https://doi.org/10.4213/tvp3827

Использование Общероссийского математического портала MathNet.Ru подразумевает, что вы прочитали и согласны с пользовательским соглашением

http://www.mathnet.ru/rus/agreement

Параметры загрузки:

IP : 3.89 .185 .249

26 апреля 2023 г., 13:34:46

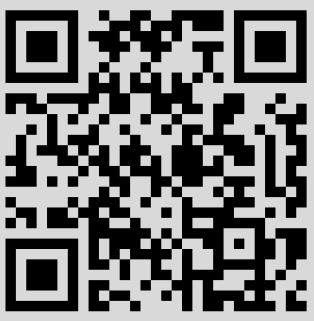


(C) 2001 г.

РОГОЗИН Б. А.*

\section{ПРЕДЕЛЬНАЯ ТЕОРЕМА ДЛЯ ОТНОШЕНИЙ ФУНКЦИЙ КОНЦЕНТРАЦИИ ${ }^{1)}$}

Доказывается предельная теорема для отношений функций концентраций сумм независимых одинаково распределенньх случайных величин.

Ключевые слова и фразы: решетчатое и нерешетчатое распределения, функция концентрации Леви, предельные теоремы для отношений.

Напомним, что невырожденное распределение $F$ с характеристической функцией $f$ нерешетчатое, если $|f(t)|<1$ при $t \neq 0$, и решетчатое с шагом $h>0$, если $|f(2 \pi k / h)|=1$ при $k=\ldots,-1,0,1, \ldots$, и $|f(t)|<1$ для остальньх значений $t$, а функция концентрации случайной величины $S_{n} Q(n, z)=\sup _{x} \mathbf{P}\left\{x \leqslant S_{n} \leqslant x+z\right\}$, где $S_{n}=X_{1}+\cdots+X_{n}$ при $n=1,2, \ldots$ и $\left\{X_{1}, X_{2}, \ldots\right\}$ - последовательность независимых случайных величин с распределением $F$. С помощью предельньх теорем для отношений вероятностей (см. $[1$, теоремы $2 \mathrm{a}, 2 \mathrm{~b}]$ ) доказывается предельная теорема для отношений функций концентрации.

Теорема. Если распределение $F$ нерешетчатое, то при фиксированньх $z_{1}>0$, $z_{2}>0 u m=0,1, \ldots$

$$
\lim _{n \rightarrow \infty} \frac{Q\left(n+m, z_{1}\right)}{Q\left(n, z_{2}\right)}=\frac{z_{1}}{z_{2}} .
$$

Если распределение $F$ решетчатое с иагом $h$, то при фиксированных $z_{1} \geqslant 0$, $z_{2} \geqslant 0 u m=0,1, \ldots$

$$
\lim _{n \rightarrow \infty} \frac{Q\left(n+m, z_{1}\right)}{Q\left(n, z_{2}\right)}=\frac{\left[z_{1} / h\right]+1}{\left[z_{2} / h\right]+1},
$$

где $[x]-$ челая часть иисла $x$.

Д о к а з а т е л ь с т в о. Для применения предельньх теорем для отношений вероятностей, установим, что при $z>0$

$$
\lim _{n \rightarrow \infty} n^{-1} \ln Q(n, z)=0 .
$$

Для этого докажем лемму.

Лемма. $\lim _{n \rightarrow \infty} \int_{0}^{1 / z}|f(t)|^{n+1} d t / \int_{0}^{1 / z}|f(t)|^{n} d t=1$. $2, \ldots$ :

Д о к а з а т е л ь с т в о. Введем последовательность плотностей $p_{n}, n=1$,

$$
p_{n}(t)=\frac{|f(t)|^{n}}{\int_{0}^{1 / z}|f(t)|^{n} d t}
$$

при $0 \leqslant t \leqslant 1 / z$ и $p_{n}(t)=0$ для остальных значений $t$. Докажем, что при любом $\varepsilon$, $0<\varepsilon<1 / z$

$$
\lim _{n \rightarrow \infty} \int_{A(\varepsilon)} p_{n}(t) d t=0
$$

где $A(\varepsilon)=[\varepsilon, 1 / z]$, если $F$ - нерешетчатое распределение, и

$$
A(\varepsilon)=\left[\varepsilon, \frac{1}{z}\right] \backslash \bigcup_{k=1}^{\infty}\left[\frac{2 k \pi}{h}-\varepsilon, \frac{2 k \pi}{h}+\varepsilon\right],
$$

* Омский филиал Института математики им. С. Л. Соболева СО РАН, ул. Певцова, 13, 644099 Омск, Россия; e-mail: rogozin@iitam.omsk.net.ru

1) Работа выполнена при поддержке Российского фонда фундаментальньх исследований (проекты 99-01-01130, 00-15-96178) и INTAS (проект 99-01317). 
если $F$ - решетчатое распределение с шагом $h$. Нетрудно заметить, что ввиду непрерывности $f$ для заданного $\varepsilon$ существует $\delta>0$ такое, что $A(\varepsilon) \subset\{t: 0 \leqslant t \leqslant 1 / z$, $|f(t)| \leqslant 1-\delta\}$. Для этого $\delta$ имеет место неравенство

$$
\int_{0}^{1 / z}|f(t)|^{n} d t \geqslant l\left(B\left(\frac{\delta}{2}\right)\right)\left(1-\frac{\delta}{2}\right)^{n},
$$

где $B(\delta / 2)=\{t: 0 \leqslant t \leqslant 1 / z,|f(t)|>1-\delta / 2\}$ и мера Лебега этого множества $l(B(\delta / 2))>0$. Следовательно, так как $|f(t)| \leqslant 1-\delta$ на $A(\varepsilon)$ и имеет место (5), то немедленно получаем соотношение (4). Из этого соотношения следует, что $\lim _{n \rightarrow \infty} \int_{0}^{1 / z}|f(t)| p_{n}(t) d t=1$. Последнее утверждение совпадает с утверждением леммы.

Из этой леммы немедленно получаем соотношение

$$
\lim _{n \rightarrow \infty} n^{-1} \ln \int_{0}^{1 / z}|f(t)|^{n} d t n^{-1}=0 .
$$

Для функций концентрации (см. [2, лемма 2.2.1]) имеют место при некоторых положительных постоянных $A_{1}$ и $A_{2}$ следующие неравенства:

$$
A_{1} z \int_{0}^{1 / z}|f(t)|^{2 n} d t \leqslant Q(n, z) \leqslant A_{2} z \int_{0}^{1 / z}|f(t)|^{n} d t .
$$

Применяя соотношение (6) к этим неравенствам, получим (3).

Если $F$ - решетчатое распределение с шагом $h$, то, поскольку функция концентрации не зависит от сдвига случайной величины, без ограничения общности можно считать, что распределение $F$ сосредоточено в точках $\{\ldots,-h, 0, h, \ldots\}$, т.е. является арифметическим с шагом $h$. Далее, выберем последовательность $\left\{x_{n}, n=1,2, \ldots\right\}$, для которой $Q(n, h / 2)=\mathbf{P}\left\{S_{n}=x_{n}\right\}$. В силу теоремы 2а [1], так как имеет место (3), для фиксированньгх $m=0,1, \ldots$ и $z>0 \lim _{n \rightarrow \infty} \mathbf{P}\left\{x_{n} \leqslant S_{n+m} \leqslant x_{n}+z\right\} / Q(n, h / 2)=$ $[z / h]+1$. Откуда вытекает, поскольку

$$
\begin{aligned}
\mathbf{P}\left\{x_{n} \leqslant S_{n+m} \leqslant x_{n}+z\right\} & \leqslant Q(n+m, z) \leqslant\left(\left[\frac{z}{h}\right]+1\right) Q\left(n+m, \frac{h}{2}\right) \\
& \leqslant\left(\left[\frac{z}{h}\right]+1\right) Q\left(n, \frac{h}{2}\right),
\end{aligned}
$$

что $\lim _{n \rightarrow \infty} Q(n+m, z) / Q(n, h / 2)=[z / h]+1 ;$ из последнего равенства следует соотношение (2).

Если $F$ - нерешетчатое распределение, то можно выбрать последовательность вещественных чисел $\left\{y_{n}, n=1,2, \ldots\right\}$ таких, что $Q(n, z)=\mathbf{P}\left\{y_{n} \leqslant S_{n} \leqslant y_{n}+z\right\}$ (см. [2, следствие теоремы 1.1.3]). Для фиксированных $m=0,1, \ldots, z>0, \alpha>0$ в силу соотношения (3) и теоремы $2 \mathrm{~b}[1]$

$$
\lim _{n \rightarrow \infty} \frac{\mathbf{P}\left\{y_{n} \leqslant S_{n+m} \leqslant y_{n}+\alpha z\right\}}{Q(n, z)}=\alpha .
$$

Откуда при $\alpha=1$, так как $Q(n+m, z) \leqslant Q(n, z)$, следует соотношение (1) при $z_{1}=$ $z_{2}=z$. Таким образом, для доказательства соотношения (1) необходимо установить, что при фиксированньг $z>0, \alpha>0$

$$
\lim _{n \rightarrow \infty} \frac{Q(n, \alpha z)}{Q(n, z)}=\alpha .
$$

Если $\alpha$ - целое число, то (8) вытекает из неравенства $Q(n, \alpha z) \leqslant \alpha Q(n, z)$ и соотношения (7). Если $\alpha=k_{1} / k_{2}$, где $k_{1}$ и $k_{2}$ - целые положительные числа, то выберем $y$ таким образом, чтобы $\alpha z=k_{1} y$ и $z=k_{2} y$, и так как $\lim _{n \rightarrow \infty}(Q(n, \alpha z) / Q(n, y))(Q(n, z) / Q(n, y))^{-1}=k_{1} / k_{2}$, то соотношение (8) доказано при рациональном $\alpha>0$. Для доказательства соотношения (8) при иррациональном $\alpha$ используем рациональные приближения для $\alpha$ и соотношение (8), доказанное для рациональньгх $\alpha$. Это завершает доказательство теоремы. 


\section{СПИСОК ЛИТЕРАТУРЫ}

1. Kesten $H$. Sums of independent random variables - without moment conditions. Ann. Math. Statist., 1972, v. 43, № 3, p. 702-732.

2. Хенгартнер В., Теодореску Р. Функции концентрации. М.: Наука, 1980, 173 с.

Поступила в редакцию 29.I.2001

(c) 2001 г.

CACOULLOS T.* , PAPADATOS N.**,
PAPATHANASIOU V.

\section{AN APPLICATION OF A DENSITY TRANSFORM AND THE LOCAL LIMIT THEOREM ${ }^{2)}$}

Рассмотрим абсолютно непрерывную случайную величину $X$ с конечной дисперсией $\sigma^{2}$. Известно, что существует другая случайная величина $X^{*}$ (которую можно рассматривать как преобразование $X$ ) с унимодальной плотностью, удовлетворяющая обобщенному ковариационному тождеству типа Стейна $\operatorname{Cov}[X, g(X)]=\sigma^{2} \mathbf{E}\left[g^{\prime}\left(X^{*}\right)\right]$ для любой абсолютно непрерывной функции $g$ с производной $g^{\prime}$, такой, что $\mathbf{E}\left|g^{\prime}\left(X^{*}\right)\right|<\infty$. С помощью этого преобразования получены верхние границы для расстояния по вариации между двумя абсолютно непрерывными случайными величинами $X$ и $Y$. В качестве приложения предлагается доказательство локальной предельной теоремы для сумм независимых одинаково распределенньх случайных величин в наиболее общей постановке.

Ключевые слова и фразы: преобразование плотности, локальная предельная теорема для плотностей, полная вариация.

1. Introduction. For an absolutely continuous (a.c.) random variable (r.v.) $X$ with density $f$, mean $\mu$, variance $\sigma^{2}$, and support $S(X)$ (through this paper we will always mean the support of an absolutely continuous r.v. $X$ with density $f$ to be the set $S(X)=$ $\{x: f(x)>0\})$, consider the r.v. $X^{*}$ with density $f^{*}$ given by the relation

$$
f^{*}(x)=\frac{1}{\sigma^{2}} \int_{-\infty}^{x}(\mu-t) f(t) d t=\frac{1}{\sigma^{2}} \int_{x}^{\infty}(t-\mu) f(t) d t .
$$

The following known properties of $X^{*}$ (see [9] or [15]) will be used in the sequel.

Lemma 1.1. (i) The density $f^{*}$ is unimodal and absolutely continuous with mode $\mu$ and maximal value $f^{*}(\mu)=\mathbf{E}|X-\mu| /\left(2 \sigma^{2}\right)$.

(ii) For any absolutely continuous function $g$ such that $\mathbf{E}\left|g^{\prime}\left(X^{*}\right)\right|<\infty$,

$$
\operatorname{Cov}[X, g(X)]=\sigma^{2} \mathbf{E}\left[g^{\prime}\left(X^{*}\right)\right] .
$$

Moreover, $X^{*}$ is characterized by this property.

* University of Athens, Department of Mathematics, Panepistemiopolis, 15784 Athens, Greece.

${ }^{* *}$ University of Cyprus, Department of Mathematics and Statistics, P. O. Box 20537, Nicosia 1678, Cyprus.

1) Part of this work was done when the author was visiting the University of Cyprus.

2) Research partially supported by the Ministry of Industry, Energy and Technology of Greece, grant 1369. 